\title{
Suitability of Neuroimaging in Diagnosis of Sleep, Fatigue, Perfusion and Metabolite Deficits after Mild Traumatic Brain Injury
}

\author{
Subhendra N Sarkar ${ }^{1 *}$, Xiang Fu Zhang ${ }^{1}$, Mary Alice Browne ${ }^{1}$ and John Claude Krusz ${ }^{2}$ \\ ${ }^{1}$ Department of Radiologic Technology \& Medical Imaging, New York City College of Techology, City University of New York, New York, USA \\ ${ }^{2}$ Anodyne Headache and Pain Care, Dallas, USA
}

*Corresponding author: Subhendra N Sarkar, Department of Radiologic Technology \& Medical Imaging New York City College of Techology, City University of New York, A414, 300 Jay St, Brooklyn, NY, USA.

Received Date: December 18,2019

Published Date: January 06, 2020

\begin{abstract}
The objective of this review is to summarize the findings of promising imaging approaches that may provide mechanistic understanding of mild traumatic brain injury (mTBI). Imaging in mTBI has so far explored brain tissue damage or physiologic changes involving perfusion and metabolite distribution. Clinical manifestations of sleep, visual disturbances and associated fatigue observed in a substantial number of mTBI patients have not been diagnosed at all. Although the association of TBI with sleep disorders is being recognized, the mechanistic relation is far from clear. The association of fatigue and sleep with mTBI raises the question which one comes first. This has been addressed to some extent in this review. Patients with mTBI and persistent post-concussive symptoms have a high incidence of medial temporal lobe injury leading to altered hippocampal, caudate and insular perfusion. The mTBI literature discusses the importance of oxidative stress and BBB disruption. Although SPECT is very sensitive in this regard, various imaging modalities with tools to map perfusion, metabolite and functional deficiencies are showing significant promise. It is a concern that MR spectroscopic abnormalities in mTBI go mostly undetected. We could not find any work that addresses secondary metabolite levels like GABA, Glutamate and so on or explores the whole brain to assess mTBI induced global metabolite abnormalities. Further mechanistic understanding to link sleep and fatigue is necessary. In conclusion, combining tissue biology to model the impact of oxidative stressor and injury localization with tracer imaging and functional connectivity mapping is preferred as well as global perfusion/metabolite measurements. Multi-modal approaches hold more promise than advanced applications of single modalities. Focal surgical procedures as in the placement of neurological shunts or neuroimplants offer excellent templates to study the deficits from static loads in pre-planned locations and may help mechanistic evaluation of mTBI that usually involves dynamic and global loads.
\end{abstract}

Keywords: Mild TBI; mTBI; Head trauma; Fatigue; Perfusion imaging; SPECT; MRI in TBI; Functional MRI in TBI; MR Spectroscopy in TBI; Metabolite imaging in TBI

Abbreviations: TBI: Traumatic Brain Injuryl; mTBI: Mild Traumatic Brain Injury; SPECT: Single Photon Emission Computed Tomography; PET: Positron Emission Tomography; MRI: Magnetic Resonance Imaging; CT: Computed Tomography; MEG: Magnetoencephalography; DTI: Diffusion Tensor Imaging (Diffusion Tractography); fMRI: Functional MRI; LOC: Loss of Consciousness; PTA: Post Traumatic-Amnesia; GSC: Glasgow Coma Scale; CDC: Center for Disease Control; PCD: Post Concussive Disorder

\section{Introduction}

Mild traumatic brain injury (mTBI) is defined by the Management of Concussion/mTBI Working Group as Normal appearance in imaging; a brief period of loss of consciousness (LOC) less than 30 minutes; post traumatic amnesia (PTA) for less than a day; and with Glasgow Coma Scale (GCS) between 13-15. The injury is usually as a result of the head being struck by an object or undergo a whiplash motion. Consistent with the prevalence of Mild
TBI, an estimation of $42 \mathrm{M}$ people worldwide and more than $1.2 \mathrm{M}$ in USA suffer from mTBI or concussion every year [1]. The exact damage it has on the brain is largely a mystery due to the lack of reliable diagnostic tools. Most patients with mTBI have impaired or absent cerebral autoregulation within 48 hours of injury [2], especially, sleep disorder, being one of the main complaints on the same day as mTBI [3]. Still neurocognitive deficits are reported 
only in $10 \%$ of cases, significantly underestimating the long-term burdens associated with it [3-6]. CDC has recently included sleep disturbances as a common effect of mTBI. The chronic stage of mTBI involves a complex and, linked/mix of symptoms including headaches, fatigue and sleep disorders for which the diagnostic and treatment options are inadequate [4].

The objective of this review is to summarize the findings of promising imaging approaches that may provide mechanistic understanding of mild traumatic brain injury (mTBI). Imaging in mTBI has so far explored brain tissue damage or physiologic changes involving perfusion and metabolite distribution. Clinical manifestations of sleep, visual disturbances and associated fatigue observed in a substantial number of mTBI patients have not been diagnosed at all. We feel that clinical manifestations of sleep, visual disturbances and associated fatigue in a substantial number of mTBI patients that do not recover fully after mTBI may now be explained by multi-modal neuroimaging that carry complementary information.

Routine clinical modalities like MRI or CT performed immediately after mTBI usually do not reveal structural damage of the brain tissues. Even when MR or CT is positive, the findings are subtle, such as petechial hemorrhage, mild edema or small contusions, considered 'minor' findings needing no neurosurgical intervention. The majority of mTBI survivors recover fully within 3-4 weeks. However, a significant minority (10-20\%) continue to experience symptoms for several months (including subacute postconcussive disorder, PCD) or often years (chronic PCD) [5]. Hence, there is a growing consensus that routine structural imaging tools do not fully reflect the underlying microscopic environment or the chronic progression of neurobiological effects of mTBI [3,6-8]. It is claimed on the other hand that, the positive predictive value of the physiologic tools like radiotracer SPECT or PET increases as mTBI progresses from acute to chronic stage and the negative predictive value also reaches nearly $100 \%$ at the chronic stage for SPECT [9]. This adds sensitivity for SPECT imaging in terms of cell membrane permeability at the level of functional blocks in vascular endothelium with impaired cerebrovascular autoregulation after TBI [10]. However, there might be other biomarkers present in mTBI to which SPECT is not differentially sensitive enough resulting in loss of specificity.

It is worth noting that analogous to minor brain trauma, when controlled, surgical intervention for placing Deep Brain Stimulation (DBS) electrodes has created increased global cerebral perfusion [11] and have been verified to produce moderately increased MR signal from whole brain tissue even under low Power MRI techniques [12]. Hence the perfusion results after minor head trauma have to be carefully assessed and global insult originating from perhaps oxidative stress model based on whiplash or dynamic blunt load should be taken into account.

Although the association of TBI with sleep disorders is being recognized, the mechanistic relation is far from clear $[5,8]$. The association of fatigue and sleep with mTBI [13] raises the question should the mTBI patients with fatigue and sleep disorders be assessed for chronic fatigue, given the prevalence of neuropsychological symptoms in many of them [14]. The conversion from acute to chronic symptoms in TBI that accompanies fatigue among young patients [15] requires further study. Similarly, the local blood-brain barrier (BBB) disruption in the microvasculature [16] and the distal spread of injury due to the cerebral vulnerability as demonstrated in minor blast-induced TBI [17] are inadequate to create a mechanistic model as claimed by radiotracer imaging [18]. Of interest to note that repetitive blast-induced injury in war zones often causes fatigue as well as sleep disorders along with long-term cognitive deficits although somewhat differently than other forms of mTBI [19].

Patients with mTBI and persistent post-concussive symptoms have a high incidence of medial temporal lobe injury leading to altered hippocampal, caudate and insular perfusion [20]. Quantification of altered hippocampal and medial temporal lobe perfusion requires sensitivity to small perfusion changes. Radiotracers like 99m-Tc work well when there exist reliable control regions in the contralateral side (internal control) or by comparing with age-matched control subjects. In routine clinical setting it is difficult to obtain baseline controls using healthy controls for isotope-based SPECT or PET, both being high radiation modalities. Radiation-free perfusion methods like arterial spin labeling MRI are promising but suffer from high inter-subject variability and low resolution [21,22].

\section{Discussion}

We have evaluated published works (from 1995- pre-prints from 2020) containing keywords such as mild traumatic brain injury, sleep disorders/issues, fatigue and neuroimaging and have identified 40 articles that have conclusively observed or discussed at least one dominant damages due to mTBI or investigated similar situations to contribute to mechanistic understanding of mTBI. The goal was to summarize common, contributory factors detectable by various modalities in a complementary fashion instead of requiring several modalities to agree on a single issue post mTBI.

Key publications in mTBI seem to stress on the importance of oxidative stress and BBB disruption. Although ECD SPECT is very sensitive for mTBI, with advancements in various imaging modalities other perfusion, metabolite and functional imaging tools could be used for early detection and treatment planning.

\section{Effects on both frontal and temporal lobes: injury translation}

The microvascular damage in mild to moderate TBI has been well staged by susceptibility weighted MRI in terms of hemorrhagic lesions commonly in frontal and temporal regions indicating local mechanical strain and microvasculature rupture. However, skull anatomy plays a significant role in translating the focal injury to distal regions by bony ridges and fasciculi [23]. Since anterior fossa structures are more mobile compared to posterior fossa structures, undersurface of the frontal and temporal lobes undergoes more 
frequent deformity when colliding with skull protuberances. Frontal and temporal lobes are interconnected by arcuate and uncinate fasciculi that often are affected by TBI. This may be the primary reason for dysfunction of both lobes and is revealed by perfusion imaging [18] (injury translation).

\section{Sleep disturbance observed across all patients}

Coup-contrecoup brain injury following mTBI usually occurs at the skull base around bony irregularities like the sphenoid ridges [24] and could explain the sleep disturbances that are observed for almost all patients in an affected mTBI group [4,5]. The inferior frontal lobe is one of the important regions involved in sleep initiation [25]. This may also indicate that not only medial temporal and frontal lobes are vulnerable in MTBI where the SPECT hypo perfusions were noted, the inferior frontal and anterior temporal regions also should be considered that are often difficult to image due to large intensity attenuation. A recent work with diffusion tensor imaging [26] found that a significant number (39\%) of patients with mTBI had diffusional changes (decreased Fractional Anisotropy) of the arcuate fasciculus although due to significant bony susceptibility in this region diffusion MRI may not be reliable.

\section{Mechanism of tracer hypoperfusion in mTBI SPECT imaging}

Microvascular membrane functions in the frontal and temporal regions change in mTBI leading to imperfect tracer retention and washout. An ideal "trapping" of 99m-Tc ECD happens if both the esterase ligands are used when ECD crosses the BBB and forms a polar ECD complex that stays away from lipid membrane and is trapped in the parenchyma [6]. TBI generates abnormal influx of $\mathrm{Ca}+2$ causing acidosis [20] with subsequent rise in oxidative stress affecting cell membrane permeability. Qualitatively, acidic environment from oxidative stress reduces esterase production and enzymatic action [6] and hence could explain the tracer hyporetention in frontal and temporal lobes in mTBI.

\section{What comes first: sleep issues or fatigue}

It is unclear what causes sleep issues. Using the same concept of oxidative stress affecting endothelial junction proteins we think that SPECT perfusion mechanics in mTBI may be due to bilateral cerebral injury seen by functional MRI [27]. None of these explain the mechanistic source of injury by itself. However, significantly elevated levels of F2-isoprostanes translated from frontal to occipital lobes may indicate late effects of oxidative stress for TBI [28], particularly when there is fatigue [13] accompanying mTBI. The sequence of oxidative stress leading to altered homeostasis in mTBI [2] might cause peroxidation of membrane lipids and generate excess intracellular calcium disrupting homeostasis and increasing membrane permeability. Since increased reactive oxidation species (ROS) in mTBI affect endothelial cells and alter cerebral autoregulation including ATP generation, a systemic fatigue often accompanying mTBI seem to get worse several months after emergence of sleep issues.

\section{Oxidative stress and effects of mTBI on endothelial tight junctions}

The blood-brain barrier consists of the cerebral microvascular endothelium, pericytes, astrocytes and neurons, out of which the endothelial changes seem to be best suited for imaging by perfusion measurements. Neuroimaging presents a unique opportunity to understand BBB disruption when mTBI precedes fatigue. Perhaps one may identify the effects of oxidative stress on the tight junctions (TJ) in vascular endothelium under three inter-dependent events: impaired autoregulation after mTBI $[10,16]$, during local ischemic injury and a widespread occurence of metabolite deficits in addition to perfusion abnormalities [28].

Endothelial junctional (tight junction, TJ) proteins play important roles in vascular permeability, and toward in-vitro stretch model of TJ proteins for mild-blast brain trauma [29]. We think the $\mathrm{TJ}$ stretch model can be applied to the increased permeability of microvasculature in the frontal and temporal lobes to explain the reduced esterase activity for ECD tracer in $\mathrm{MTBI}$ just because there may be greater Red-ox activity post mTBI in this region) [18]. The effects of biaxial stretch were characterized at 5, 10, 15, 25, and 50\% deformation (mild to moderate in trauma scale in the stretch model) that induced a deformation-dependent increase in LDH release, cell death, and activation of caspase $3 / 7$, suggesting the induction of apoptosis. For mTBI with small deformations, an increase in TJ proteins is expected as a compensation mechanism [29] that might generate imperfect "polar" ECD forms in the vulnerable, frontal temporal lobes enabling ECD to early washout [18].

\section{mTBI at chronic phase: utility of multimodal approach}

If and when the effects from mTBI enters chronic phase more resources need to be allocated, due to additional complexity, to diagnose and treat to preserve quality of life and offer suitable preventive treatment. As MRI begins to look at the acute to chronic transition, routine clinical imaging perhaps should be avoided. However, a recent fMRI work to detect fatigue in patients with previous TBI [27] has demonstrated altered striato-thalamiccortical functioning in caudate and anterior thalamus. Multimodal brain imaging may start with a sensitive tool like SPECT followed by a non-invasive research tool like resting state connectivity fMRI or Magnetoencephalography or MEG to detect altered memory and executive functions [30]. MRI based diffusion tractography [31] and neurometabolite spectroscopy [32,33] could also advance the understanding of chronic components. There is a growing consensus [34] that an effective treatment plan would benefit from combined structural, physiologic and functional assessments both at acute and at chronic phases [35].

\section{Conclusion}

This review emphasizes the following three main findings of imaging in mTBI:

A. Mild traumatic brain injury causes mechanical damage that often indirectly leads to perfusion heterogeneity in the 
frontal and temporal lobes and perhaps over time by oxidative stressor or vascular effects translate the injury to occipital lobe.

B. Deficits in the occipital region, not observed by routine MRI or nuclear SPECT or PET contribute to visual problems and perhaps contribute to sleep disturbances. It is not clear if this can change the levels of major metabolites like NAA and be observed by sampling basal ganglia or occipital lobe using MR spectroscopy. A slight reduction of NAA in the occipital lobe has been observed [32] as well as diffusion tract changes [31]. It is also not known if metabolites move around or replenished as some of the mTBI patients recover.

C. Deficits in the thalamus region bordering the frontal lobe are common for mTBI as often seen by isotope imaging in SPECT or PET $[18,32]$ which is an important region of the brain that helps promote sleep and maintain wakefulness.

It is worth noting that MR spectroscopic abnormalities in mTBI are mostly absent $[32,36]$. However, this needs to be critically evaluated and confirmed. Mild Trauma and subsequent global oxidative stress could alter Glutamate pathways, particularly if fatigue is to be explained. Measuring major metabolites alone, particularly by localized single voxel measures, seem to be insufficient to conclude about the utility of spectroscopy. One may use the whole brain approach using 3D multi-voxel spectroscopy with emphasis in limbic region and assess with GABA and Glutamate detection to observe patterns of secondary metabolite imbalance in the affected group. To our knowledge, it is not clear if neuro metabolite levels alter over time when mTBI progresses in certain patient groups and get replenished in others. Particular attention may be given for metabolite follow ups in frontal, temporal and occipital regions due to early sleep and visual disturbances presumably originating in basal ganglia and tentorium.

Further mechanistic understanding to link sleep and fatigue is necessary. Also combining tissue biology to model impact of oxidative stressor and injury localization with tracer imaging and functional connectivity mapping [30] could be performed as well as perfusion/metabolite measurements. Multi-modal approaches hold more promise than advanced applications of single modalities like repeat CT for Hounsfield unit changes [37] or susceptibility MR for micro-hemorrhages [33] or PET/SPECT with new tracer designs. Repetitive minor blast trauma [17] as well as focal surgical procedures as in the placement of neurological shunts or implants $[11,12]$ may act as templates to study effects of controlled, static loads in pre-planned locations to offer useful mechanistic evaluation of mTBI that usually involves dynamic and global loads.

\section{Acknowledgement}

One of the authors (X.Z.) obtained a scholarship from City University of New York under Emerging Scholars program to work on the mTBI project.

\section{Conflict of Interest}

The authors do not have any direct or indirect conflict of interest.

\section{References}

1. Leo P and McCrea M (2016) Epidemiology. In: Laskowitz D, Grant G (Eds.), Translational research in traumatic brain injury. CRC Press/ Taylor \& Francis Group, Boca Raton, FL, USA.

2. Junger EC, Newell DW, Grant GA, Avellino AM, Ghatan S, et al. (1997) Cerebral autoregulation following minor head injury. J Neurosurg 86: 425-432.

3. https://www.cdc.gov/traumaticbraininjury/mtbi_guideline.html.

4. Krusz JC (2009) Post-traumatic headaches, migraines, and sleep disorders. Pract Pain Manage 9:70-81.

5. Sigurdardottir S, Andelic N, Roe C, Jerstad T, Schanke AK (2009) Postconcussion symptoms after traumatic brain injury at 3 and 12 months post-injury: a prospective study. Brain Injury 23: 489-497.

6. Gowda NK, Agrawal D, Bal C, Chandrashekar N, Tripati M, et al. (2006) Technetium Tc-99m Ethyl Cysteinate Dimer brain Single-Photon Emission CT in mild traumatic brain injury: A prospective study. Am J Neuroradiol 27: 447-451.

7. Bigler ED, Maxwell WL (2012) Neuropathology of mild traumatic brain injury: Relationship to neuroimaging findings. Brain Imaging Behav 6: 108-136.

8. Wickwire EM, Williams SG, Roth T, Capaldi VF, Jaffe M et al. (2016) Sleep, sleep Disorders, and mild traumatic brain injury. What we know and what we need to know: Findings from a national working group. Neurotherapeutics 13: 403-417.

9. Raji CR, Tarzwell R, Pave D, Schneider H, Uszler M et al. (2014) Clinical utility of SPECT neuroimaging in the diagnosis and treatment of traumatic brain injury: A systematic review. PLoS ONE 9: e91088.

10. Salehi A, Zhang JH, Obenaus A (2017) Response of the cerebral vasculature following traumatic brain injury. J Cereb Blood Flow Metab 37: 2320-2339.

11. Sidtis JJ, Tagliati M, Alterman R, Sidtis DVL, Dhawan V, et al. (2012) Therapeutic high frequency stimulation of the subthalamic nucleus in Parkinson's disease produces global increases in cerebral blood flow. J Cereb Blood Flow Metab 32: 41-49.

12. Sarkar S, Alterman R, Pavassiliou E, Rojas R (2016) Low Power MRI Techniques for Neurosurgical Planning and Post-Surgical Assessment of Deep Brain Stimulators in Patients with Medically Refractory Parkinson's Disease or Dystonia. IOSR Journal of Dental and Medical Sciences 15: 71-75.

13. Englander J, Busnik T, Oggins J, Katznelson L. (2010) Fatigue after traumatic brain injury: association with neuroendocrine, sleep, depression and other factors. Brain Injury 24:1379-88.

14. Mahmood O, Rapport LJ, Hanks RA, Fichtenberg NL (2004) Neuropsychological performance and sleep disturbance following traumatic brain injury. J Head Trauma Rehab 19: 378-390.

15. Kamper JE, Pop V, Fukuda A, Ajao D, Hartman R et al. (2013) Juvenile traumatic brain injury evolves into a chronic brain disorder: Behavioral and histological changes over 6 months. Exp Neurol 250: 8-19.

16. Logsdon AF, Lucke-Wold BP, Turner RC, Huber JD, Rosen CL, et al. (2015) Role of microvascular disruption in brain damage from traumatic brain injury. Compr Physiol 5: 1147-1160.

17. Kamnaksh A, Ahmed F, Kovesdi E, Barry ES, Grunberg NE, et al. (2014) Molecular mechanisms of increased cerebral vulnerability after repeated mild blast-induced traumatic brain injury. Translational Proteomics 3: 22-37.

18. Crider T, Eng D, Sarkar PR, Cordero J, Krusz JC, et al. (2018) Microvascular and large vein abnormalities in young patients after mild head trauma and associated fatigue: A brain SPECT evaluation and posture dependence modeling. Clin Neurol Neurosurg 170: 159-164.

19. Jason LA, Najar N, Porter N, Reh C (2009) Evaluating the Centers for Disease Control's empirical chronic fatigue syndrome case definition. Journal of Disability Policy Studies 20: 93-100. 
20. Umile EM, Sandel ME, Alavi A, Terr CM, Plotkin RC (2002) Dynamic imaging in mild traumatic brain injury: Support for the theory of medial temporal vulnerability. Arch Phys Med Rehabil 83: 1506-1513.

21. Li X, Spence JS, Sarkar SN, Metzger GJ, Haley RW, etal. (2012) Hippocampal longitudinal sub-region perfusion can be reliably measured using ASL. Proc. 20th Annual Meeting ISMRM, Melbourne, Australia, \#722.

22. Li X, Sarkar SN, Purdy DE, Spence JS, Haley RW, et al. (2013) Anteroposterior perfusion heterogeneity in human hippocampus measured by arterial spin labeling MRI. NMR Biomed 26: 613-621.

23. Bigler ED. (2007) Anterior and middle cranial fossa in traumatic brain injury: relevant neuroanatomy and neuropathology in the study of neuropsychological outcome. Neuropsychology 21: 515-531.

24. Viola-Saltzman M, Watson NF (2012) Traumatic brain injury and sleep disorders. Neurol Clin 30: 1299-1312.

25. Ayalon L, Borodkin K, Dishon L, Kanety H, Dagan Y (2007) Circadian rhythm sleep disorders following mild traumatic brain injury. Neurology 68: $1136-1140$

26. Jang SH, Lee AY, Shin SM (2016) Injury of the arcuate fasciculus in the dominant hemisphere in patients with mild traumatic brain injury: A retrospective cross-sectional study. Medicine (Baltimore) 95: e3007.

27. Berginström N, Nordström P, Ekman U, Eriksson J, Andersson M, et al. (2018) Using functional magnetic resonance imaging to detect chronic fatigue in patients with previous traumatic brain injury: changes linked to altered striato-thalamic-cortical functioning. J Head Trauma Rehabil 33: $266-274$.

28. Fontana L, Giagulli C, Minuz P, Lechi A, Laudanna C (2001) 8-Iso-PGF2 alpha induces beta 2-integrin-mediated rapid adhesion of human polymorphonuclear neutrophils: a link between oxidative stress and ischemia/reperfusion injury. Arterioscler Thromb Vasc Biol 21: 55-60.
29. Rosas-Hernandez H, Cuevas E, Escudero-Lourdes C, Lantz SM, GomezCrisostomo NP, et al. (2018) Characterization of biaxial stretch as an in vitro model of traumatic brain injury to the blood-brain barrier. Mol Neurobiol 55: 258-266.

30. McDonald BC, Saykin AJ, McAllister TW (2012) Functional MRI of mild traumatic brain injury (mTBI): progress and perspectives from the first decade of studies. Brain Imaging Behav 6: 193-207.

31. Khong E, Odenwald N, Hashim E, Cusimano MD (2016) Diffusion tensor imaging findings in post-concussion syndrome patients after mild traumatic brain injury: a systematic review. Front Neurol 7: 156.

32. Brown M, Baradaran H, Christos PJ, Wright D, Gupta A, et al. (2018) Magnetic resonance spectroscopic abnormalities in traumatic brain injury: A meta-analysis. J Neuroradiology 45: 123-129.

33. Lewine JD, Davis JT, Bigler ED, Thoma R, Hill D, et al. (2007) Objective documentation of traumatic brain injury subsequent to mild head trauma: Multimodal brain imaging with MEG, SPECT, and MRI. J Head Trauma Rehabil 22: 141-155.

34. Krusz JC, Mears FG and Katz HN (2015) Traumatic brain injury: Part 3: Evaluation, treatment, and rehabilitation. Pract Pain Manage 15: 46-56.

35. Eierud C, Craddock RC, Fletcher S, Aulakh M, King-Casas B, et al. (2014) Neuroimaging after mild traumatic brain injury: review and metaanalysis. Neuroimage Clin 4: 283-294.

36. Kubas B, Lebkowski W, Lebkowska U, Kułak W, Tarasow E, et al. (2010) Proton MR spectroscopy in mild traumatic brain injury. Polish J Radiol 75: 7-10.

37. Almenawer SA, Bogza I, Yarascavitch BK, Reddy K (2013) The value of scheduled repeat cranial computed tomography after mild head injury. Neurosurg 72: 56-64 